\title{
Pediatric neuromuscular disorders: Care considerations during the COVID-19 pandemic
}

\author{
Anne Troike Stratton ${ }^{\mathrm{a}, *}$, Richard Ogden Roberts III $^{\mathrm{b}}$, Oren Kupfer ${ }^{\mathrm{c}}$, Terri Carry ${ }^{\mathrm{d}}$, Julie Parsons ${ }^{\mathrm{e}}$ and \\ Susan Apkon ${ }^{\mathrm{f}}$ \\ ${ }^{a}$ Department of Physical Medicine and Rehabilitation, Children's Hospital Colorado, University of Colorado School \\ of Medicine, Aurora, CO, USA \\ ${ }^{\mathrm{b}}$ Department of Pediatrics, Section of Diabetes and Endocrinology, Texas Children's Hospital, Baylor College of \\ Medicine, Houston, TX, USA \\ ${ }^{\mathrm{c}}$ Department of Pediatrics, Section of Pulmonary Medicine, Children's Hospital Colorado, University of Colorado \\ School of Medicine, Aurora, CO, USA \\ ${ }^{\mathrm{d}}$ Children's Hospital Colorado, University of Colorado School of Medicine, Aurora, CO, USA \\ ${ }^{\mathrm{e}}$ Department of Pediatrics, Section of Neurology, Haberfeld Family Endowed Chair in Pediatric Neuromuscular \\ Disorders, Children's Hospital Colorado, University of Colorado School of Medicine, Aurora, CO, USA \\ ${ }^{\mathrm{f}}$ Department of Physical Medicine and Rehabilitation, Fischahs Chair in Pediatric Rehabilitation, Children's \\ Hospital Colorado, University of Colorado School of Medicine, Aurora, CO, USA
}

\begin{abstract}
COVID-19, the respiratory and frequently systemic disease caused by the novel SARS-COV-2 virus, was first recognized in December 2019 and quickly spread to become a pandemic and world-wide public health emergency over the subsequent 3-4 months. While COVID-19 has a very low morbidity rate across approximately $80 \%$ of the population, it has a high morbidity and mortality rate in the remaining $20 \%$ of the population. ${ }^{1}$ These numbers have put a significant strain on medical systems around the world. Patients with neuromuscular diseases such as those with Duchenne muscular dystrophy (DMD) and spinal muscular atrophy (SMA), tend to be more medically fragile and have higher health care needs than the general population. Respiratory insufficiency, cardiac disease, obesity, and immunocompromised status due to chronic steroid treatments in certain patient populations with neuromuscular conditions are specific risk factors for severe COVID-19 disease. In general, the pediatric population has shown to be less severely impacted with lower infection rates and lower morbidity and mortality rates than the adult population, however, as expected, children with underlying medical conditions are at higher risk of morbidity from COVID-19 than their peers. ${ }^{2}$ Many patients with neuromuscular disease also rely heavily on caregiver support through their lifetime and thus maintaining the health of their primary caregivers is also a significant consideration in the health and well-being of the patients. This paper will address routine and emergency medical care, rehabilitation services, and other considerations for the pediatric patient with a neuromuscular condition during the COVID-19 pandemic.
\end{abstract}

Keywords: Duchenne muscular dystrophy and COVID-19, SARS-CoV-2 and muscular dystrophy, rehabilitation and COVID-19, spinal muscular atrophy and COVID-19, telehealth and muscular dystrophy, teletherapy

\footnotetext{
${ }^{*}$ Corresponding author: Anne Troike Stratton, Department of Physical Medicine and Rehabilitation, University of Colorado School of Medicine, Children's Hospital Colorado 13123 E 16th Ave, B 285, Anschutz Medical Campus, Aurora, CO 80045, USA. Tel.: +1 720 777 2806; E-mail: anne.stratton@childrenscolorado.org.

${ }^{1}$ Wu Z, McGoogan JM. Characteristics of and Important Lessons from the Coronavirus Disease 2019 (COVID-19) Outbreak in China:
}

\footnotetext{
Summary of a Report of 72âẮr 314 Cases from the Chinese Center for Disease Control and Prevention. JAMA. 2020; 323(13): 1239-1242. doi: 10.1001/jama.2020.2648.

${ }^{2}$ Viner RM, Whittaker E. Kawasaki-like disease: emerging complication during the COVID-19 pandemic. Lancet. 2020; 395(10239): 1741-1743. doi: 10.1016/S0140-6736(20)31129-6.
} 


\section{Introduction}

COVID-19, the respiratory and frequently systemic disease caused by the novel SARS-COV-2 virus, was first recognized in December 2019 and quickly spread to become a pandemic and world-wide public health emergency over the subsequent 3-4 months. While COVID-19 has a very low morbidity rate across approximately $80 \%$ of the population, it has a high morbidity and mortality rate in the remaining $20 \%$ of the population [1]. These numbers have put a significant strain on medical systems around the world.

The virus is transmitted primarily via air-born droplets [2] and an infected host can shed and spread it for several days while asymptomatic [3]. No degree of herd immunity exists to this virus at the time of the writing of this paper making it particularly hard to contain, though distancing, isolation, careful hygiene, and widespread personal protection equipment use such as face masks has helped to decrease the current viral effective reproduction number [4].

Patients with neuromuscular diseases such as those with Duchenne muscular dystrophy (DMD) and spinal muscular atrophy (SMA), tend to be more medically fragile and have higher health care needs than the general population. Respiratory insufficiency, cardiac disease, obesity, and immunocompromised status due to chronic steroid treatments in certain patient populations with neuromuscular conditions are specific risk factors for severe COVID-19 disease. In general, the pediatric population has shown to be less severely impacted with lower infection rates and lower morbidity and mortality rates than the adult population. However, as expected, children with underlying medical conditions are at higher risk of morbidity from COVID-19 than their peers [5]. Many patients with neuromuscular disease also rely heavily on caregiver support through their lifetime and thus maintaining the health of their primary caregivers is also a significant consideration in the health and well-being of the patients.

Fortunately, data gathered thus far, while limited, has shown that neuromuscular patient populations have not had high morbidity and mortality from COVID-19 at this point. It may be that they and their caregivers have been successful in avoiding exposure to the virus with current distancing measures, or that they have not been getting sick enough to require testing and hospitalization. A recent survey conducted from early April through May 11, 2020 by Cure SMA (pending publication) reported that only four patients with spinal muscular atrophy out of over 1,000 patients and fami- lies surveyed had been diagnosed with COVID-19 and only 1 of those patients had required hospitalization to date [6]. While this is reassuring, risk from this virus remains high until a safe and effective vaccine is widely available and distributed.

This paper will address routine and emergency medical care, rehabilitation services, and other considerations for the pediatric patient with a neuromuscular condition during the COVID-19 pandemic.

\section{COVID-19 presentation and morbidity}

COVID-19 has a broad range of symptoms as have been fairly well described including fever, cough, myalgias, fatigue, shortness of breath, diarrhea, and loss of smell and taste $[7,8,10]$. Severe illness manifests as pneumonia, acute respiratory failure, acute respiratory distress syndrome (ARDS), and may also be associated with acute liver injury, acute cardiac injury, secondary infection, acute kidney injury, septic shock, disseminated intravascular coagulation, blood clots, rhabdomyolysis and Multisystem Inflammatory Syndrome in Children (MIS-C). [1,8,8,9] MIS-C is a newly described condition with similarities to Kawasaki disease that has been linked to SARS-COV-2 virus exposure in children [5]. Incidence rate, risk factors and other epidemiologic data is emerging, but it is unclear at this time whether any characteristics of certain neuromuscular diseases affect risk for this still rare immune-mediated sequelae.

Comorbidities associated with increased disease severity and higher mortality include obesity, hypertension, cardiovascular disease, chronic lung disease, chronic kidney disease, liver disease, smoking, and cancer, as well as advanced age. [8-10] Severe morbidity and increased mortality outcomes also have been shown to disproportionately affect racial and ethnic minorities, likely related to societal conditions that lead to later presentation to care, decreased access to health care, underlying health conditions, lack of paid sick leave, multigenerational households, and increased exposure to the virus while being in close proximity or living with an essential worker [11,12].

These factors should all be taken into consideration when assessing risk and discussing mitigation strategies against contracting SARS-COV-2 in patients with neuromuscular conditions. 


\section{Medical management during a pandemic}

\subsection{General medical considerations}

Given that patients with neuromuscular disease in general have a higher reliance on medical equipment, medications, home care and medical supplies as well as caregiver support, every patient and family should have and review an emergency plan. Supply chains may be affected, home nursing and therapies may be disrupted, and caregivers may be vulnerable to illness or burn out with less community support available. Extra supplies and medications should be secured, and plans should be made for back up caregivers to be trained and available to help if needed. Telehealth appointments should be considered for routine medical appointments if possible. In some instances, telehealth may be a valuable tool to help triage whether an in-person appointment is needed in the event of a non-urgent medical issue.

Patients with neuromuscular disease often have cardiac and respiratory compromise including cardiomyopathy, restrictive lung disease, impaired cough function, aspiration, and sleep-disordered breathing. They may rely on medical technology (non-invasive or invasive ventilation, mechanical in-exsufflation, suction, inhaled medications, etc.) to maintain respiratory health $[13,14]$. Patients with acute cardiorespiratory symptoms, fever, or increased respiratory secretions, thought secondary to COVID-19 or another viral illness, should be evaluated in a health care facility capable of managing their complex condition. If this is not possible, the evaluating provider should contact the patient's neuromuscular team for assistance and to discuss the respiratory care plan. The patient and caregivers should bring their home equipment and settings to any urgent or scheduled visit to eliminate treatment lag time in set up and fitting equipment and accessories.

Initial evaluation should include vital signs and a complete physical examination including thorough cardiorespiratory exam. Diagnostic testing should include chest X-ray, blood gas, and evaluation of electrolytes and renal function. Of note, renal function is often overestimated by serum creatinine values in patients with muscle disease. Therefore, a careful assessment of fluid status is needed, and providers should consider nonroutine renal function tests such as cystatin C. In patients with cardiac disease, an electrocardiogram (ECG) should be obtained. Patients with neuromuscular disease, especially SMA, are at risk for hypoglycemia during prolonged fasting. Therefore, measurement of serum glucose and initiation of a supportive glucose infusion rate is recommended.

\subsection{Respiratory care}

Patients with neuromuscular disease can have a range of respiratory function, from normal to severe restriction and chronic respiratory failure. COVID-19 risk stratification by baseline lung function parameters or other functional parameters has not been established. However, those patients with a forced vital capacity less than $50 \%$ predicted, impaired cough function, or established use of noninvasive ventilation or augmented airway clearance, should be considered most at risk for acute respiratory failure, intubation, and death from any respiratory tract infection $[13,15]$. Patients with mild-to-moderate restrictive lung disease (FVC \% predicted $>50 \%$ and less than $80 \%$ ) or with moderately diminished cough function should also be considered at risk for severe respiratory disease as respiratory muscle load is increased during times of physiologic stress and can result in rapid deterioration $[13,14]$. Patients with episodic or waxing/waning diseases like some congenital myasthenic syndromes and myasthenia gravis may have normal lung function when well but can have profound changes in respiratory muscle function in response to respiratory infections [16]

Assessing risk of respiratory failure in infants and children too young or too impaired to perform pulmonary function tests is challenging. Underlying diagnosis (including but not limited to spinal muscular atrophy types 1 and 2, congenital myotonic dystrophy, severe congenital myopathies, severe congenital muscular dystrophies, and mitochondrial myopathies) can imply high risk of acute respiratory failure [17]. Prior use of noninvasive ventilation, sleep-disordered breathing at baseline, impaired cough function, use of augmented cough devices at baseline, a history of respiratory failure or hospitalization with respiratory infections, or prolonged intubation after anesthesia are historical clues that should prompt aggressive interventions with noninvasive ventilation and augmented cough function. Whenever possible, consultation with the patient's neuromuscular team, especially the pulmonologist and respiratory therapist, is advised.

Respiratory care should focus on airway clearance and assistive ventilation. Biphasic noninvasive ventilation to support respiratory effort and airway clearance aimed at augmenting cough function are the mainstays of respiratory support and should be used early in acute disease $[13,14,18,19]$. In children and adults with neuromuscular disease, hypoxemia is often a sign of hypoventilation. Patients may not display respiratory distress because of weak respiratory muscles. Therefore, 
a high index of suspicion is required to assess patients adequately.

In patients with known restrictive lung disease (FVC $<80 \%$ predicted), inspiratory muscle weakness (maximal inspiratory pressure $<60 \mathrm{~cm} \mathrm{H}_{2} \mathrm{O}$ ), chronic hypoventilation, or sleep-disordered breathing, biphasic noninvasive ventilation should be used early in illness $[13,14]$. This modality should be used even in the absence of acute hypoventilation on blood gas analysis in order to support respiratory muscle function and reduce the risk of acute respiratory failure caused by increased respiratory muscle load and metabolic demand. Supplemental oxygen, high flow nasal cannula oxygen, and continuous positive airway pressure (CPAP) are not appropriate treatments for patients with neuromuscular disease $[13,14]$. Patients with previously normal lung function should be evaluated carefully as acute illness can exacerbate muscle weakness and lead to acute respiratory failure. Supplemental oxygen may be used in conjunction with biphasic noninvasive ventilation once adequate ventilation is achieved. Increases in the fraction of inspired oxygen $\left(\mathrm{FiO}_{2}\right)$ suggest mucus obstruction, atelectasis, worsening lower respiratory tract disease, or worsening hypoventilation. Therefore, providers should consider adjustment of noninvasive ventilation settings to address hypoxemia.

In some cases, intubation with mechanical ventilation is indicated. Medical providers should discuss this with patients or their families as many have advance directives. The primary neuromuscular team that knows the patient well should be involved in this discussion when possible. While an acute illness such as COVID19 may be treatable with invasive mechanical support, the underlying neuromuscular condition may not permit extubation after a significant illness and so the team should discuss the possibility of tracheostomy.

Airway clearance should focus on augmenting impaired cough function [13,14,17-19]. In adolescents and adults, a peak cough flow less than $160 \mathrm{~L} / \mathrm{min}$ when well is associated with impaired mucus clearance [15]. A peak cough flow between 160 and $270 \mathrm{~L} / \mathrm{min}$ is associated with increased risk of impaired cough function during illness and these patients should receive aggressive airway clearance [20]. Normative data for peak cough flows do not exist in younger children. Historical signs of impaired cough function should be investigated. Mechanical augmentation of cough function is usually achieved with mechanical in-exsufflation, commonly referred to as cough assist. This device can be used with a variety of interfaces including mouthpiece, facemask, and endotracheal tube. Hypoxemia is often a result of mucus obstruction and should be treated with aggressive airway clearance. An experienced pulmonologist or respiratory therapist is vital to achieving best results. Mechanical in-exsufflation can also prevent failures of extubation [21]. Airway clearance is sometimes augmented with inhaled medications (bronchodilators or mucolytics) $[22,23]$ or other mechanical treatment such as a vibration vest [24,25] or intrapulmonary percussive ventilation [26,27]. These may be added to mechanical in-exsufflation and noninvasive ventilation on an as needed basis but are not sufficient alone [13,14,18,19].

Because SARS-CoV-2, the virus that causes COVID19 disease, is spread by airborne droplets $[2,3]$ and invasive and noninvasive ventilation and mechanical airway clearance are thought to increase the possibility of generating infectious droplets, some healthcare facilities have attempted to limit the use of these therapies. This is not appropriate for patients with neuromuscular disorders and can lead to prolonged respiratory illness, more severe illness, and death. Neuromuscular advocacy groups have made recommendations and many healthcare facilities have adjusted their practice by utilizing various strategies such as filters, personal protective equipment (PPE), and isolation practices to permit these respiratory therapies while reducing risk of aerosolization contamination ${ }^{1}$ Patients and parents should advocate for these practices.

\subsection{Acute management considerations for patients on steroids}

Daily glucocorticoid therapy remains the mainstay of treatment for DMD to improve and prolong muscle strength and cardiac and pulmonary function [28,29]. Steroid treatment is indicated in all stages of the disease and thus often prescribed for many years, spanning a patient's life, decades from the time of initial prescription [30]. Additionally, glucocorticoid therapy may be trialed in a variety of other neuromuscular diagnoses [31]. Patients receiving onasemnogene aboparvovec gene transfer therapy for SMA are treated with oral prednisolone one day prior to dosing and for at least two months after treatment. Treatment with chronic systemic steroids, however, is known to cause significant multi-system side effects, including but not limited to, suppression of endogenous adrenal function with

\footnotetext{
${ }^{1}$ www.curecmd.org/covid19-resources, www.parentprojectmd.org/ care/ppmd-covid-19-coronavirus-information-center/, www.curesma. org/covid19/.
} 
subsequent adrenal insufficiency and immunosuppression [32,33]. As such, the Center for Disease Control and Prevention (CDC) currently lists individuals on chronic corticosteroid therapy as one of a number of high risk groups for the development of severe disease from COVID-19 infections. However, the immediate discontinuation of chronic steroid therapy is not a feasible option for most individuals due to ongoing benefits of therapy and risk for sequela of stopping after chronic use. [29,34]. Therefore, important considerations must be made in guiding ongoing corticosteroid therapy in patients with neuromuscular disease with or without active SARS-CoV-2 infection [30,35].

Many organizations, including the CDC, the Endocrine Society, the Muscular Dystrophy Association, and Parent Project Muscular Dystrophy, advise patients with neuromuscular conditions treated with chronic steroid therapy to not abruptly cease their regular medications [30,35]. Discontinuation of therapy may precipitate an adrenal crisis, a life-threatening medical emergency due to deficiency of cortisol, the body's endogenous glucocorticoid, in the absence of exogenous steroids [34]. The PJ Nicholoff Steroid Protocol was developed specifically to address such concerns in males with Duchenne and Becker muscular dystrophy [30]. Adrenal crises in patients with suppressed adrenal function typically occurs in the setting of physiologic stressors such as a febrile illness, which is likely to be seen with COVID-19 infections [32]. Patients with neuromuscular conditions receiving chronic glucocorticoid therapy should be taught stress dose precautions including when and how to increase their steroids as well as how to administer an intramuscular injection of hydrocortisone (Solucortef ${ }^{\circledR}$ ) to mitigate the risk of decompensation in the setting of COVID-19 should they become ill [34]. Both the current clinical care guidelines for the management of DMD and the PJ Nicholoff Steroid Protocol detail typical dosing strategies [29,30]. If hospitalized due to severe illness, patients' home steroids should be converted to hydrocortisone to cover their stress dose needs and to reduce the risk of adverse outcomes of high dose corticosteroid therapy, although data in pediatric patients with COVID-19 are lacking [36]. Consultation with a pediatric endocrinologist should be considered [35].

\subsection{Issues around receiving novel treatments for SMA and DMD during a pandemic}

For those living with neuromuscular conditions, the treatment landscape is changing with many novel ther- apies in clinical trial and some receiving FDA approval $[37,38]$. For providers delivering care to individuals being treated with one of these approved drugs, it is important to be familiar with the dosing schedules, potential challenges if doses are missed, and considerations for resuming treatments that may be put on hold. Planning for these instances will improve care delivery.

Exon skipping drugs including eteplirsen and golodirsen $[39,40]$ used to treat boys with DMD are given as weekly intravenous infusions either in the hospital, infusion center, or at home in most circumstances. Individual cases should be reviewed to determine the safest scenario for patients and their families to limit exposure to SARS-CoV-2. For home therapy, there should be systems in place whereby home health providers are screened and supplied with appropriate PPE prior to giving infusions. To further limit exposure and excess patient clinic visits, safety labs can be drawn by home health providers as well.

Patients with SMA currently have two FDA approved therapies available [41]. Onasemnogene abeparvovec (gene transfer therapy) is approved for children under the age of two years and nusinersen is for patients of all ages with $5 \mathrm{q}$ SMA. Both of these treatments require administration at a health care facility. Depending on the facility, SARS-CoV-2 testing prior to any treatment or procedure may be required. Especially for newly diagnosed patients, there is urgency to initiate treatment quickly as several studies have shown that earlier treatment leads to improved outcomes $[42,43]$. Many states now have newborn screening programs in place resulting in identification of presymptomatic infants who may derive significant benefit if treated in the first weeks of life. Treatment with either of these agents is critical to the health of patients with SMA and should not be considered optional or elective and thus subject to delays.

Onasemnogene abeparvovec is administered through a peripheral IV. Patients are treated with oral prednisolone one day prior to dosing and for at least two months after treatment. They need close clinical and laboratory follow up for thrombocytopenia, troponin and elevated liver enzymes during this period of time. Throughout the period of immunosuppression, family members and care providers should closely follow recommended COVID-19 guidelines regarding hand washing and social distancing measures. Consideration should be given to use of telehealth appointments when appropriate as well as home health visits for laboratory assessments. As stated earlier, appropriate PPE and COVID-19 safeguards must be in place for any home health monitors. 
Nusinersen is delivered via intrathecal injection. Four loading doses are given during the first two months of treatment and quarterly thereafter for life. Lumbar punctures may be done in an office, procedure center, or an interventional radiology suite depending on the patient's clinical status and degree of spinal deformity. Anesthesia for the procedure may be required which increases risk for both the patient and medical team. If a dose is missed or delayed, nusinersen package insert instructions advise treating as quickly as possible to maintain appropriate cerebrospinal fluid concentration. Safety labs for coagulopathies and urine protein are monitored prior to dosing.

\section{Rehab management during a pandemic}

The COVID-19 pandemic has created considerable challenges in providing rehabilitation services to our patients with neuromuscular conditions. It has also created some unique opportunities for creative management of these patients through telerehabilitation and other resources. Most in-person visits for multi-disciplinary clinics and home, clinic or school- based therapies have either been cancelled or rescheduled as telehealth depending on the local capabilities and local and state regulatory requirements.

Standards of care for management of most if not all neuromuscular disorders recommend frequent, standardized assessments of function in a multidisciplinary clinic or care center as well as ongoing therapy services at home or in the community [29,44]. Meeting these standards of care becomes complex and challenging during "stay at home" recommendations and each situation should be carefully evaluated as to how to best meet the needs of the patient. These needs are also complicated by the many other challenges facing caregivers as they try to balance work from home or in some cases loss of employment, care for their children at home with online/distance learning and telehealth visits for therapies and other services. This exhausting and stressful schedule can be hard to manage and should be taken into consideration by all providers making recommendations for ongoing care.

\subsection{Telehealth utilization for clinical assessments}

Regular assessments, typically every 6 months, of patients with neuromuscular conditions such as spinal muscular atrophy and Duchenne muscular dystrophy are recommended for most neuromuscular diagnosis.
Telerehabilitation can give providers the opportunity to observe the patients in their natural environment and in many cases provides a window into the resources as well as limitations to patient's care at home. Outcome measurement assessments administered by telerehabilitation cannot be done in the standardized manner in which they were designed in most cases. Modified versions of these assessments, however, can be done by using a little creativity and can provide useful information on current status and help in making recommendations for therapy interventions. For example, many household items such as quarters, plastic containers with lids, printer paper, and soup cans can be used to substitute for standardized items in the Performance of Upper Limb [45] used for patients with Duchenne/Becker muscular dystrophy and the Revised Upper Limb Module [46], used for patients with SMA. The Northstar Ambulatory assessment (NSAA) [47], is a useful tool for measuring ambulatory function in Duchenne and many other neuromuscular diagnoses. Most of the items in this assessment can easily be administered during a telehealth visit with some exceptions and modifications. Similarly, many items on the CHOP INTEND [48], Hammersmith Functional Motor Scale Expanded (HFMSE) [49] and Revised Hammersmith scale (RHS) [50] can also be done with some limitations and modifications via telehealth. Accurate comparisons to past testing results are not possible and a statement to that effect should be noted in the medical record. Timed function testing cannot be done due to time delays on video and range of motion measurements cannot be accurately administered via telehealth. However, useful information on current function can be obtained from these modified assessments and can help guide home therapy and exercise recommendations.

It is important to note that because assessments cannot be accurately compared to previous assessments, they cannot be used for clinical trial exams. International academic networks are currently working to develop, standardize and validate telehealth (telerehabilitation) assessments.

Additionally, prior to authorizing approval or reapproval for reimbursement for novel treatments such as eteplirsen, golodirsen, or nusinersen, many insurance companies require standardized testing. Care providers may need to proactively discuss these challenges with payors. Functional assessments required in clinical trials will vary depending on the sponsor, local or central IRB requirements, and the hospital in which they are performed. Research therapists should work closely with the Principal Investigator and Research Coordi- 
nator to assure adherence to the study protocol in the setting of the pandemic.

Adaptive equipment management can be performed with some limitations if an in-person visit is not possible. In other cases, equipment that is not typically available for in-clinic visits can be evaluated via telehealth. This is a great way to assess fit, proper use and safety of home equipment. Standers, walkers, sitting devices, backup wheelchairs or power chairs that can't be transported can be seen for perhaps the first time. Families can be encouraged to share their creative home "inventions" both for function and recreation thereby providing caregivers and therapists an opportunity to learn and share with others. Durable medical equipment providers may be available for in-person visits or telehealth to make necessary repairs or to assist with the face-to-face assessments that are often required. Providers and therapists should collaborate with them to address patient needs as quickly as possible.

Orthotics should be assessed for fit during each visit whether in person or by telehealth. If any pressure areas are detected, the patient should be directed to contact their orthotist. If modifications cannot be made due to visitation restrictions, avoiding use should be advised until these modifications can be done.

\subsection{Home exercise and therapy program considerations}

Providing therapy is typically hands-on and finding ways to make this work remotely can dramatically change the way it is performed. However, integrating therapy into virtual visits is possible and can be quite helpful. Telerehabilitation may be provided by community, school or clinic therapists. Scheduling telehealth therapy visits should be coordinated with the multiple other virtual appointments a child may have with online learning, IEP meetings and visits with medical providers. The therapist providing care remotely takes on more of a teaching rather than touching role and has the responsibility to guide the parents in the activities that are recommended. Coordinating and sharing goals between therapists is essential for continuity of care and to create shared goals. Setting expectations appropriately while keeping in mind the limitations and stress the caregivers might be experiencing is important to consider.

Continued use of night splints, standers and daily stretching and positioning should be recommended for most patients especially if the child is receiving more limited therapy services [51] and may be more helpful if incorporated into a daily routine [51]. During the COVID-19 pandemic, the parent or caregiver has been forced to assume the sole responsibility for providing all the in-person therapy services that were previously provided by the therapists.

Encouraging physical activity during the pandemic is recommended as it is useful in preventing disuse atrophy and contractures [29,44]. Creative suggestions are needed when social distancing guidelines are in place and may include: a walk around the block, a family bike ride, use of a stander while playing a game, hand or leg ergometers, massage, dance parties, virtual yoga or Pilates, pool therapy in the bathtub, bubbles or even meditation for deep breathing.

In summary, rehabilitation assessments and therapy interventions can and should be modified through telerehabilitation and telephone encounters when inperson visits are not possible. Individualized plans of care should be developed to meet the needs and challenges of each patient.

\section{School impacts of a pandemic}

The transition to remote learning for children and families during the COVID-19 pandemic was abrupt and without adequate resources in many situations [52]. While the effect on all children is not known at this time, one can presume that children with disabilities were likely negatively impacted. In Section 504 of the Rehabilitation Act of 1973 and the Individuals with Disabilities Education Act (IDEA) of 1975, children with disabilities were guaranteed educational opportunities that supported their unique learning needs [53]. Specialized learning services, nursing services, therapy services, technology, equipment, and other accommodations provide children the ability to learn and thrive in the school setting. As the pandemic forced children to remain at home, those with disabilities lost the infrastructure required to be successful.

Families and educators, faced with remote learning for the child with a disability, require creative problemsolving to address the multitude of challenges. Internet access must be available and accessible for the child to transition to remote learning and therefore children in households with fewer resources are placed at greater risk for falling behind [54]. For those with severe physical impairments, specialized computer interfaces may be necessary and available only in the school environment thereby limiting a child's ability to participate in educational opportunities from home. Telepractice can 
connect children with therapy team members in order to carry out their Individualized Educational Plan (IEP) in the home setting. However, the amount of therapy services may be more limited during periods of remote learning, placing the responsibility of supplementing these services on the parent or caregiver who will have limited expertise in administering the therapy. Additional mental health supports may be necessary for children and caregivers placed under new stressors with reduced peer interaction.

As school districts develop reopening plans, parents, therapists, and healthcare providers need to advocate for a safe and effective return. Guidance from the Centers for Disease Control and Prevention is general and without specific or clear guidelines for children with disabilities [55]. A balance between safety of the child and caregivers and the benefits of returning to school must be struck with special attention to planning for social distancing, the challenges of some children tolerating a face covering, and cleaning specialized equipment. In accordance with IDEA, children with disabilities should return to the school in the least restrictive environment. Re-evaluations by therapists and learning specialists should be completed at the start of the schoolyear to understand the current level of functioning of the child, recognizing the potential for loss of skills during the stay at home period and summer break during the pandemic. IEP's should be reviewed as proactively as possible, with modifications made as needed. To remain in compliance with IDEA, schools need to assure that the IEP meets the child's "unique needs and prepares them for further education, employment, and independent living." During the COVID-19 pandemic, these plans require attention to the physical, learning, and psychosocial needs of the child [56].

\section{Conclusion}

The COVID-19 pandemic has placed unprecedented pressures on our modern health care systems. This has resulted in rapid changes to health care delivery and unique stressors on patients with neuromuscular conditions. Patients with neuromuscular conditions and their caregivers have experienced new levels of hope with recent scientific advances in care in the past several years but the worry of COVID-19 has threatened this. In summary, patients, caregivers, and medical providers need to consider the higher health care needs, higher level of caregiver dependence, dependence on ventilatory support technology, higher incidence of chronic steroid use, need for timely access to medical treatments, need for physical and other therapy modalities, and unique school accommodation requirements affected by COVID-19. Despite the fears and new stress caused by the pandemic, with careful adherence to public health recommendations, careful management of acute medical issues, and use of telehealth technology, patients with neuromuscular conditions can continue high level medical and rehabilitation care to remain healthy and thrive.

\section{Acknowledgments}

The authors have no funding sources or other acknowledgments to disclose. They appreciate the support and expertise of their colleagues, trainees, and staff of the multidisciplinary neuromuscular clinic at Children's Hospital Colorado.

\section{Conflict of interest}

Julie Parsons, MD is the principal investigator on clinical trials for PTC Therapeutics, Novartis Gene Therapies, Biogen, and Scholar Rock and has received consulting fees from Novartis Gene Therapies, Biogen, and Sarepta Therapeutics.

Susan Apkon, MD has received consulting fees from Biogen and Sarepta Therapeutics.

The other authors have no conflicts or financial interests to report.

\section{References}

[1] Wu Z, McGoogan JM. Characteristics of and Important Lessons from the Coronavirus Disease 2019 (COVID-19) Outbreak in China: Summary of a Report of 72314 Cases from the Chinese Center for Disease Control and Prevention. JAMA. 2020; 323(13): 1239-1242. doi: 10.1001/jama.2020.2648.

[2] Anderson EL, Turnham P, Griffin JR, Clarke CC. Consideration of the Aerosol Transmission for COVID-19 and Public Health. Risk Anal. 2020; 40(5): 902-907. doi: 10.1111/risa.13500.

[3] Yu X, Yang R. COVID-19 transmission through asymptomatic carriers is a challenge to containment [published online ahead of print, 2020 Apr 4]. Influenza Other Respir Viruses. 2020; doi: doi10.1111/irv.12743,

[4] Bruinen de Bruin Y, Lequarre AS, McCourt J, et al. Initial impacts of global risk mitigation measures taken during the combatting of the COVID-19 pandemic [published online ahead of print, 2020 Apr 15]. Saf Sci. 2020; 128: 104773. doi: 10.1016/j.ssci.2020.104773. 
[5] Viner RM, Whittaker E. Kawasaki-like disease: emerging complication during the COVID-19 pandemic. Lancet. 2020; 395(10239): 1741-1743. doi: 10.1016/S0140-6736(20)311296.

[6] CureSMA Coronavirus; (COVID-19) Community Webinar: Latest and Next Steps, May 28, 2020.

[7] Veerapandiyan A, Wagner KR, Apkon S, McDonald CM, Mathews KD, Parsons JA, et al. The care of patients with Duchenne, Becker, and other muscular dystrophies in the COVID-19 pandemic. Muscle Nerve. 2020. doi: 10.1002/mus. 26902.

[8] Richardson S, Hirsch JS, Narasimhan M, Crawford JM, McGinn T, Davidson KW and the Northwell COVID-19 Research Consortium. Presenting Characteristics, Comorbidities, and Outcomes Among 5700 Patients Hospitalized With COVID-19 in the New York City Area. JAMA. 2020; 323(20): 2052-2059. doi: 10.1001/jama.2020.6775.

[9] Cummings MJ, Baldwin MR, Abrams D, O’Donnell MR, et al. Epidemiology, clinical course, and outcomes of critically ill adults with COVID-19 in New York City: a prospective cohort study. Lancet. 2020 Jun 6; 395(10239): 1763-1770. doi: 10.1016/S0140-6736(20)31189-2.

[10] Huang C, Wang Y, Li X, Ren L, Zhao J, Wang J, Cao B, et al. Clinical features of patients infected with 2019 novel coronavirus in Wuhan, China. Lancet. 2020; 395: 497-506. doi: 10.1016/S0140-6736(20)30183-5

[11] Kirby T. Evidence mounts on the disproportionate effect of COVID-19 on ethnic minorities. Lancet Respir Med. 2020 Jun; 8(6): 547-548. doi: 10.1016/S2213-2600(20)30228-9.

[12] Garg S, Kim L, Whitaker M, et al. Hospitalization Rates and Characteristics of Patients Hospitalized with LaboratoryConfirmed Coronavirus Disease 2019 - COVID-NET, 14 States, March 1-20, 2020. Morb Mortal Wkly Rep. 2020; 69: 458-464.

[13] Birnkrant DJ, Bushby K, Bann CM, et al. Diagnosis and management of Duchenne muscular dystrophy, part 2: respiratory, cardiac, bone health, and orthopaedic management. Lancet Neurol. 2018; 17(4): 347-361. doi: 10.1016/S14744422(18)30025-5

[14] Finkel RS, Mercuri E, Meyer OH, et al. Diagnosis and management of spinal muscular atrophy: Part 2: Pulmonary and acute care; medications, supplements and immunizations; other organ systems; and ethics. Neuromuscul Disord. 2018; 28(3): 197-207. doi: 10.1016/j.nmd.2017.11.004.

[15] Bach JR, Saporito LR. Criteria for extubation and tracheostomy tube removal for patients with ventilatory failure. A different approach to weaning. Chest. 1996; 110(6): 15661571. doi: 10.1378/chest.110.6.1566.

[16] Kinali M, Beeson D, Pitt MC, Jungbluth H, Simonds AK, et al. Congenital myasthenic syndromes in childhood: diagnostic and management challenges. J Neuroimmunol. 2008; 201-202: 6-12. doi: 10.1016/j.jneuroim.2008.06.026.

[17] Buu MC. Respiratory complications, management and treatments for neuromuscular disease in children. Curr Opin Pediatr. 2017; 29(3): 326-333. doi: 10.1097/MOP.0000000000 000498 .

[18] Wang CH, Bonnemann CG, Rutkowski A, Sejersen T, Bellini J, Battista V, et al. International Standard of Care Committee for Congenital Muscular Dystrophy. Consensus statement on standard of care for congenital muscular dystrophies. J Child Neurol. 2010; 25(12): 1559-1581. doi: 10.1177/ 0883073810381924.

[19] Wang CH, Dowling JJ, North K, et al. Consensus statement on standard of care for congenital myopathies. J Child Neurol. 2012; 27(3): 363-382. doi: 10.1177/0883073812436605.

[20] Tzeng AC, Bach JR. Prevention of pulmonary morbidity for patients with neuromuscular disease. Chest. 2000; 118(5): 1390-1396. doi: 10.1378/chest.118.5.1390.

[21] Bach JR, Sinquee DM, Saporito LR, Botticello AL. Efficacy of mechanical insufflation-exsufflation in extubating unweanable subjects with restrictive pulmonary disorders. Respir Care. 2015; 60(4): 477-483. doi: 10.4187/respcare.03584.

[22] Inoue D, Furubayashi T, Ogawara K, et al. In vitro evaluation of the ciliary beat frequency of the rat nasal epithelium using a high-speed digital imaging system. Biol Pharm Bull. 2013; 36(6): 966-973. doi: 10.1248/bpb.b12-01076.

[23] Boek WM, Graamans K, Natzijl H, van Rijk PP, Huizing EH Nasal mucociliary transport: new evidence for a key role of ciliary beat frequency. Laryngoscope. 2002; 112(3): 570-573. doi: 10.1097/00005537-200203000-00029.

[24] Yuan N, Kane P, Shelton K, Matel J, Becker BC, Moss RB. Safety, tolerability, and efficacy of high-frequency chest wall oscillation in pediatric patients with cerebral palsy and neuromuscular diseases: an exploratory randomized controlled trial. J Child Neurol. 2010; 25(7): 815-821. doi: 10.1177/088307380 9350223.

[25] Lechtzin N, Wolfe LF, Frick KD. The Impact of highfrequency chest wall oscillation on healthcare use in patients with neuromuscular diseases. Ann Am Thorac Soc. 2016; 13(6): 904-909. doi: 10.1513/AnnalsATS.201509-597OC.

[26] Toussaint M, De Win H, Steens M, Soudon P. Effect of intrapulmonary percussive ventilation on mucus clearance in Duchenne muscular dystrophy patients: a preliminary report. Respir Care. 2003; 48(10): 940-947.

[27] Reardon CC, Christiansen D, Barnett ED, Cabral HJ. Intrapulmonary percussive ventilation vs incentive spirometry for children with neuromuscular disease. Arch Pediatr Adolesc Med. 2005; 159(6): 526-531. doi: 10.1001/archpedi.159.6.526.

[28] Griggs RC, Miller JP, Greenberg CR, et al. Efficacy and safety of deflazacort vs prednisone and placebo for Duchenne muscular dystrophy. Neurology. 2016; 87(20): 2123-2131.

[29] Birnkrant DJ, Bushby K, Bann CM, et al. Diagnosis and management of Duchenne muscular dystrophy, part 1: diagnosis, and neuromuscular, rehabilitation, endocrine, and gastrointestinal and nutritional management. Lancet Neurol. 2018; 17(3): 251-267.

[30] Kinnett K, Noritz G. The PJ Nicholoff Steroid Protocol for Duchenne and Becker Muscular Dystrophy and Adrenal Suppression. PLoS Curr. 2017 Jun 27; 9: doi: 10.1371/currents.md.d18deef7dac96ed135e0dc8739917b6e.

[31] Bromberg MB, Carter O. Corticosteroid use in the treatment of neuromuscular disorders: empirical and evidence-based data. Muscle Nerve. 2004; 30(1): 20-37. doi: 10.1002/mus.20075.

[32] Bowden SA, Henry R. Pediatric Adrenal Insufficiency: Diagnosis, Management, and New Therapies. Int J Pediatr. 2018; 2018: 1739831. doi: 10.1155/2018/1739831.

[33] Woods CP, Argese N, Chapman M, et al. Adrenal suppression in patients taking inhaled glucocorticoids is highly prevalent and management can be guided by morning cortisol. Eur J Endocrinol. 2015; 173(5): 633-642. doi: 10.1530/EJE-15-0608.

[34] Bowden SA, Connolly AM, Kinnett K, Zeitler PS. Management of Adrenal Insufficiency Risk After Long-term Systemic Glucocorticoid Therapy in Duchenne Muscular Dystrophy: Clinical Practice Recommendations. J Neuromuscul Dis. 2019; 6(1): 31-41. doi: 10.3233/JND-180346.

[35] Kaiser UB, Mirmira RG, Stewart PM. Our Response to COVID-19 as Endocrinologists and Diabetologists. J Clin En- 
docrinol Metab. 2020; 105(5): dgaa148. doi: 10.1210/clinem/ dgaa148.

[36] Tang C, Wang Y, Lv H, Guan Z, Gu J. Caution against corticosteroid-based COVID-19 treatment. Lancet. 2020; 395(10239): 1759-1760. doi: 10.1016/S0140-6736(20)307492.

[37] Mendell JR, Al-Zaidy S, Shell R, Arnold WD, Rodino-Klapac LR, Prior TW, et al. Single-Dose Gene-Replacement Therapy for Spinal Muscular Atrophy. N Engl J Med. 2017; 377(18): 1713-22. doi: 10.1056/NEJMoa1706198.

[38] Shieh PB. Emerging Strategies in the Treatment of Duchenne Muscular Dystrophy. Neurotherapeutics. 2018; 15(4): 840-8. doi: 10.1007/s13311-018-00687-z.

[39] Frank DE, Schnell FJ, Akana C, El-Husayni SH, Desjardins CA, Morgan J, et al. Increased dystrophin production with golodirsen in patients with Duchenne muscular dystrophy. Neurology. 2020; 94(21): e2270-e82. doi: 10.1212/WNL.000 0000000009233.

[40] Heo YA. Golodirsen: First Approval. Drugs. 2020; 80(3): 32933. doi: 10.1007/s40265-020-01267-2.

[41] Finkel RS, Mercuri E, Darras BT, Connolly AM, Kuntz NL, Kirschner J, et al. Nusinersen versus Sham Control in InfantileOnset Spinal Muscular Atrophy. N Engl J Med. 2017; 377(18) 1723-32. doi: 10.1056/NEJMoa1702752.

[42] Dangouloff T, Servais L. Clinical Evidence Supporting Early Treatment Of Patients With Spinal Muscular Atrophy: Current Perspectives. Ther Clin Risk Manag. 2019; 15: 1153-61. doi: 10.2147/TCRM.S172291.

[43] De Vivo DC, Bertini E, Swoboda KJ, Hwu WL, Crawford TO, Finkel RS, et al. Nusinersen initiated in infants during the presymptomatic stage of spinal muscular atrophy: Interim efficacy and safety results from the Phase 2 NURTURE study. Neuromuscul Disord. 2019; 29(11): 842-56. doi: 10.1016/j.nmd. 2019.09.007.

[44] Mercuri E, Finkel RS, Muntoni F, Wirth B, Montes J, Main $M$, et al. Diagnosis and management of spinal muscular atrophy: Part 1: Recommendations for diagnosis, rehabilitation, orthopedic and nutritional care. Neuromuscul Disord. 2018; 28(2): 103-15. doi: 10.1016/j.nmd.2017.11.005.

[45] Mayhew A, Mazzone ES, Eagle M, Duong T, Ash M, Decostre V, et al. Development of the Performance of the Upper Limb module for Duchenne muscular dystrophy. Dev Med Child Neurol. 2013; 55(11): 1038-45. doi: 10.1111/dmcn.12213.

[46] Mazzone E, Bianco F, Martinelli D, Glanzman AM, Messina $\mathrm{S}$, De Sanctis R, et al. Assessing upper limb function in nonambulant SMA patients: development of a new module. Neuromuscul Disord. 2011; 21(6): 406-412. doi: 10.1016/j. nmd.2011.02.014.
[47] Mayhew AG, Cano SJ, Scott E, Eagle M, Bushby K, Manzur A, et al. Detecting meaningful change using the North Star Ambulatory Assessment in Duchenne muscular dystrophy. Dev Med Child Neurol. 2013; 55(11): 1046-52. doi: $10.1111 / \mathrm{dmcn} .12220$

[48] Glanzman AM, Mazzone E, Main M, Pelliccioni M, Wood J, Swoboda KJ, et al. The Children's Hospital of Philadelphia Infant Test of Neuromuscular Disorders (CHOP INTEND): test development and reliability. Neuromuscul Disord. 2010; 20(3): 155-61. doi: 10.1016/j.nmd.2009.11.014.

[49] Glanzman AM, O'Hagen JM, McDermott MP, Martens WB, Flickinger J, Riley S, et al. Validation of the Expanded Hammersmith Functional Motor Scale in spinal muscular atrophy type II and III. J Child Neurol. 2011; 26(12): 1499-1507. doi: 10.1177/0883073811420294.

[50] Ramsey D, Scoto M, Mayhew A, Main M, Mazzone ES Montes J, et al. Revised Hammersmith Scale for spinal muscular atrophy: A SMA specific clinical outcome assessment tool. PLoS One. 2017; 12(2): e0172346. doi: 10.1371/journal.pone. 0172346.

[51] Skalsky AJ, McDonald CM. Prevention and management of limb contractures in neuromuscular diseases. Phys Med Rehabil Clin N Am. 2012; 23(3): 675-87. doi: 10.1016/j.pmr.2012. 06.009 .

[52] Viner RM, Russell SJ, Croker H, Packer J, Ward J, Stansfield $\mathrm{C}$, et al. School closure and management practices during coronavirus outbreaks including COVID-19: a rapid systematic review. Lancet Child Adolesc Health. 2020; 4(5): 397-404. doi: 10.1016/S2352-4642(20)30095-X.

[53] Lipkin PH, Okamoto J, Council on Children with D, Council on School H. The Individuals With Disabilities Education Act (IDEA) for Children With Special Educational Needs. Pediatrics. 2015; 136(6): e1650-62. doi: 10.1542/peds.20153409.

[54] Armitage R, Nellums LB. Considering inequalities in the school closure response to COVID-19. Lancet Glob Health. 2020; 8(5): e644. doi: 10.1016/S2214-109X(20)30116-9.

[55] Prevention CDCa. Coronavirus Disease 2019 (COVID-19) Childcare, Schools, and Youth Programs, Plan, Prepare, and Respond: Considerations for Schools [Internet]. 2020. Available from: https://www.cdc.gov/coronavirus/2019-ncov/ community/schools-childcare/schools.html.

[56] Education UDo. Individuals with Disabilties Education Act [internet]. [cited US Department of Education 6-5-2020]. Available from: https://sites.ed.gov/idea/about-idea/\#IDEAPurpose. 